\title{
Los campos de investigación como objeto de indagación: reflexiones teórico-metodológicas a partir de estudio de caso
}

The fields of research as an object of inquiry: theoretical-methodological reflections from a case study

Alejandra Ramos

Universidad de Buenos Aires, Facultad de Filosofia y Letras,

Instituto de Ciencias Antropológicas, Sección Etnohistoria. CONICET, Argentina

alejandraramos_hotmail.com

\section{Resumen:}

En este artículo presento reflexiones teórico-metodológicas sobre el estudio de la producción y circulación del conocimiento científico. La propuesta se desprende de una investigación de mi autoría en donde reconstruí el devenir de la Etnohistoria andina entre 1970 y 2005 . Me detengo en el enfoque y los materiales de análisis empleados, indicando los principales desafíos que se presentaron en la construcción del objeto y en el abordaje del problema de investigación. Desarrollo las estrategias implementadas y argumento que su relevancia trasciende el caso de estudio. Tras indicar los resultados obtenidos presento las líneas de indagación que se abren a partir del trabajo realizado.

Palabras Clave: Interdisciplina, Metodología, Estudios Sociales de la Ciencia, Etnohistoria , Redes académicas.

\section{Abstract:}

In this article I present theoretical and methodological reflections on the study of the production and circulation of scientific knowledge. The proposal is derived from an investigation of my authorship where I reconstructed the evolution of the Andean ethnohistory between 1970 and 2005. I describe the approach and the materials used, indicating the main challenges that emerged in the construction of the object and the research problem. I develop the strategies implemented and I argue that their relevance transcends the case study. I present the results obtained and then the lines of research that are opened from them.

KEYWORDS: Interdisciplinary, Methodology, Social Studies of Science, Ethnohistory, Academic networks.

\section{INTRODUCCIÓN}

Este trabajo se encuentra dirigido principalmente a investigadores interesados en el estudio de la producción y la circulación del conocimiento científico a través de la reconstrucción y el análisis del desarrollo de disciplinas o campos disciplinares.

En mi investigación doctoral estudié la conformación y el desarrollo de la Etnohistoria andina, desde su consolidación en la década de 1970 y hasta el 2005, siguiendo las redes académicas y producciones asociadas en tres países: Perú, Bolivia y Argentina. Los objetivos de esta investigación fueron diseñados con el espíritu de analizar la producción etnohistórica en el período señalado. A medida que la indagación avanzaba, se hizo evidente que no era sencillo precisar qué es producción etnohistórica y qué no; qué investigadores pueden ser considerados etnohistoriadores, e incluso la existencia misma de la Etnohistoria andina. A partir de interrogarme qué se consideraba lícitamente producción etnohistórica, y qué lugares y quienes eran considerados actores relevantes de este campo, orienté mi problema hacia la comprensión de las condiciones de posibilidad de la Etnohistoria andina como una forma específica de conocimiento. Identificar estas condiciones implicó considerar las cambiantes circunstancias sociopolíticas, la infraestructura empleada y creada por los investigadores, y los sentidos que estos le daban a la Etnohistoria andina en los distintos momentos históricos que aborda la tesis. Estos interrogantes desplazaron la atención hacia factores que 
requirieron el empleo de materiales de análisis disímiles y la integración de distintas perspectivas, con su consecuente desafío de pertinencia y coherencia.

En este artículo presento una serie de decisiones tomadas en el curso de la investigación y los resultados obtenidos con base en ellas. Considero que un examen reflexivo de estas decisiones puede resultar de interés a otros investigadores, ya que, si bien se desprenden del estudio de un caso, lo trascienden debido al tono teórico metodológico de la exposición. El foco estará, por lo tanto, en las decisiones tomadas y en sus resultados, y no en el análisis de los materiales, aunque indicaré en cada caso las publicaciones en las cuales el lector podrá encontrar un desarrollo pormenorizado al respecto.

En los dos primeros apartados expongo el punto de partida de la investigación: una pregunta que se originó en la antropología y lo que esto implicó en términos de abordaje. Además, planteo por qué la definición de etnohistoria andina resulta problemática y qué desafíos plantea. El tercer apartado está dedicado a explicitar los criterios de selección de los materiales de análisis en función de lo desarrollado en los dos apartados precedentes. Luego procedo a sintetizar los resultados de la investigación indicando de qué manera la construcción del objeto de estudio realizada permitió avanzar hacia una mejor comprensión de la etnohistoria andina. Finalmente, sintetizo la propuesta y expongo líneas de indagación futura que se desprenden del enfoque implementado.

\section{LA ANTropología como marco de LA PREgUNTA}

Gadamer sostiene que el lugar desde el que se pregunta conlleva "una fijación expresa de los presupuestos que están en pie y desde los cuales se muestra la cantidad de duda que queda abierta" (Gadamer, 1988, p. 441). En distintos sentidos la Antropología se constituyó como el punto de partida de mis preguntas. La investigación que realicé para mi tesis doctoral se radicó en la sección Etnohistoria del Instituto de Ciencias Antropológicas (ICA) de la Facultad de Filosofía y Letras (UBA); esto la enmarcó en dos debates actuales que perfilaron las preguntas iniciales. El primero, no del todo explicitado pero con una reconocida historia al interior del ICA, se refiere a si la Etnohistoria forma o no parte de la tradición disciplinar de la Antropología, y el segundo, más manifiesto y sobre el que abunda la bibliografía, trata sobre la vigencia actual de la Etnohistoria. Volveré sobre este punto en el siguiente apartado, cuando discuta las dificultades que tuve para la definición de mi objeto de estudio. Además, desde mi propia formación como antropóloga, me pregunté cuál podía ser -si es que lo había- el aporte de esta disciplina a los estudios sobre la producción y circulación del conocimiento científico.

Las diferentes trayectorias de la Antropología se han pensado en términos de su relación con la construcción de nación y de imperio (Stocking, 1982), a partir de estilos nacionales (Gerholm y Hannerz, 1982), como antropologías del sur (Krotz, 1997), centrales versus periféricas (Cardoso de Oliveira, 1999/2000), o hegemónicas y no hegemónicas, y luego, también, disidentes (Ribeiro y Escobar, 2008; Restrepo, 2012). Ribeiro y Escobar (2008, p. 11) argumentan que, si bien durante el siglo XX las transformaciones en la Antropología estuvieron relacionadas al sujeto/objeto de estudio, en las últimas décadas éstas se encuentran vinculadas a "las relaciones entre antropólogos ubicados en diferentes partes del sistema-mundo".

Estas investigaciones se han realizado más en paralelo que en diálogo con los estudios sociales de las ciencias. Filosofía, Sociología e Historia cuentan con una amplia tradición en lo que respecta a estudios sobre la producción de conocimiento científico. Se reconoce una consolidación del campo hacia inicios del siglo XX, y un punto de quiebre en la década de 1970, con el mayor diálogo entre Sociología e Historia, que habría permitido en la década siguiente la superación de la división de larga data entre externalismo e internalismo (Moro Abadía, 2005). Esto alentó una perspectiva que contemplaba el lenguaje a través del cual se expresaban las ideas y las condiciones histórico-sociales, institucionales y materiales dentro de las cuales -y con las cualesse producían. De esta manera, las condiciones de enunciación se entienden como parte integral del sentido 
del discurso, y las formaciones discursivas se presentan como contingentes, situadas históricamente (Palti, 2007; Primas, 2007).

En los años 80 la Antropología comenzó a tener una mayor presencia en este tipo de investigaciones, principalmente a través de su metodología con el trabajo de campo en laboratorios. Aunque pueden considerarse como antecedentes los estudios sobre pensamiento primitivo, racionalidad y magia, lo cierto es que el interés específico de la Antropología por la ciencia y la tecnología se inicia con las etnografías en laboratorios. Esto coincide con los desarrollos de una antropología del presente o de los mundos contemporáneos (Hidalgo y Stagnaro, 2016). Si bien esta línea de trabajo no tuvo en términos generales un mayor impacto en los propios estudios disciplinares de la Antropología, lo cierto es que ambos coinciden luego de retomar la propia tradición disciplinar- en poner el foco en las practicas concretas de investigación (Hidalgo y Stagnaro, 2016; Restrepo, 2012). Poner en primer plano la diversidad de las prácticas y la variabilidad de la experiencia de los investigadores implica una impronta etnográfica que puede ser replicada para pensar el pasado, orientando la mirada hacia el modo en que circulan las ideas, los contextos en que se generan y cómo son apropiadas y transformadas en el proceso de traslación a otros contextos (Gil, 2016)

Arêas Peixoto (2008) invita a pensar el aporte específico de la Antropología a partir de su presupuesto epistemológico: el conocimiento se produce en el diálogo entre puntos de vista diferentes, el de un otro y el propio. De esta manera, si seguimos a la autora, un abordaje antropológico contribuye también a superar la distinción entre una historia externa (el contexto) y una interna (el contenido), ya que

...cuando se trata de las ideas y del pensamiento, considerar seriamente el punto de vista del "otro" exige el tratamiento analítico minucioso de sus contenidos sustantivos -y del lenguaje en el que se expresa-, que no son ajenos a los contextos en los que son gestados (Arêas Peixoto, 2008, p. 22).

Las disciplinas que conforman los estudios sociales de la ciencia se encuentran en diálogo desde hace varias décadas, y esto ha contribuido a que los límites entre cada una se tornen borrosos. Sin embargo, es desde su especificad que han contribuido al desarrollo del campo ( $c f r$. Moro Abadía, 2005). Es en este sentido que me interesó identificar cuáles eran las principales propuestas desde la Antropología y cómo dialogaron o no con los estudios de las ciencias desde otros enfoques. A partir de este examen me propuse recuperar en mi propia investigación el foco en las prácticas, la identificación de experiencias diversas, la importancia de la circulación y su incidencia en la transformación de las propuestas y el carácter dialógico de la producción del conocimiento, entendiéndolos como constitutivos de un enfoque proveniente de la Antropología.

Sumada a ello, la investigación se constituyó tomando distancia de aproximaciones que circunscribieran el objeto de estudio a un lugar delimitado por fronteras claras y predeterminadas. En ese sentido, es tributaria tanto de los enfoques multisituados de la Etnografía como de los abordajes históricos que se alejan del nacionalismo metodológico. Finalmente, la articulación de los enfoques historicistas y presentistas, en términos de Stocking (1982), evita el olvido del contexto de enunciación y la propia historicidad del lugar desde el que se pregunta (la pertenencia profesional y de formación ya indicada), a la vez que nos resguarda de emitir juicios sobre las producciones analizadas desde los intereses actuales: las discusiones centradas en la crisis de la Etnohistoria, que veremos a continuación. ${ }^{1}$

\section{Problemas de definición}

Parte constitutiva del desarrollo de la investigación científica comprende la transformación progresiva de la problemática inicial a través de la identificación de sentidos y relaciones no anticipados, que conducen a la revisión de las propias categorías. Por supuesto, la pregunta inicial se formula a partir de algún conocimiento previo. En este caso, ese conocimiento consistía fundamentalmente lo expuesto en mi tesis de licenciatura, en la cual había comparado las publicaciones de dos de los tres autores consagrados -por sus propios colegas-como referentes fundacionales de la Etnohistoria andina (Ramos, 2011). ${ }^{2}$ Por otra parte, a partir de las lecturas, contaba con cierta cronología básica: la Etnohistoria andina se iniciaba en la década 
de 1970; había tenido un periodo de auge hasta fines los años 80, y, en la década siguiente, había entrado en una crisis que tuvo como resultado su extinción. Los motivos podían diferir, pero en general se asociaban o bien a que la Etnohistoria había sido una moda pasajera, o bien a que se trataba de investigaciones inherentemente esencialistas que ya no tenían lugar.

Entonces, ¿cómo seguir el desarrollo de la Etnohistoria andina a partir de los años 90 si ésta había dejado de existir? ¿Había delimitado mal la temporalidad de la investigación? Una serie de encuentros y una imagen, que parecían quedar fuera de la cronología, me impulsaron a mantener el período bajo análisis hasta el 2005. En primer lugar, los congresos internacionales de Etnohistoria (eminentemente andinos), que habían comenzado a realizarse en 1989 y que se mantienen hasta la actualidad, y, en segundo lugar, una fotografía tomada en 1997 que decoraba la oficina de la directora de mi lugar de trabajo, en donde podía verse a los tres "padres" de la Etnohistoria andina (Murra, Rowe y Zuidema) rodeados de casi dos decenas de investigadores que, de diversas maneras, se encontraban vinculados a la Etnohistoria.

Para superar esta aparente paradoja de ausencia/presencia debí alejarme de las definiciones normativas de la Etnohistoria andina y, luego de recuperar las propuestas presentadas en el apartado anterior, centrarme en las prácticas investigativas. Pensar en prácticas situadas y no en identidades trascendentes es una premisa ya asentada en los estudios antropológicos, que sin embargo no siempre se aplica en las investigaciones sobre la propia disciplina (Restrepo 2012). Tomé esta propuesta para pensar la Etnohistoria andina, con el agregado de que, en este caso, al no estar cimentado su carácter como disciplina, lo difuso y arbitrario de las delimitaciones se hacía más evidente.

Es necesaria una breve digresión antes de retomar el hilo de la exposición. Los fundamentos a partir de los cuales sostengo que la Etnohistoria andina no puede definirse inequívocamente como una disciplina han sido presentados en un trabajo previo (Ramos, 2016a). Sería demasiado extenso desarrollarlos aquí, aunque sí me gustaría señalar algunos de los puntos más salientes. La Etnohistoria surge como una las articulaciones entre Antropología e Historia que se concretaron a partir de las demandas del mundo de la segunda posguerra enmarcadas en un proceso de descolonización. Sus distintas variantes responden a áreas de investigación (norteamericana, mesoamericana, andina, europea, africana) y no a escuelas o academias nacionales. A lo largo de la segunda mitad del siglo XX se producen una serie debates que atraviesan a la Etnohistoria en sus distintas variantes: a) se discuten las definiciones que parten del objeto y asignan a la Etnohistoria el estudio de los grupos étnicos precoloniales y coloniales; b) se producen propuestas contrapuestas que la sitúan como disciplina, subdisciplina (en algunos casos de la Antropología y en otros de la Historia), espacio interdisciplinar e incluso antidisciplinar; c) se intenta fundamentar su especificidad desde lo metodológico con caracterizaciones luego discutidas por su vaguedad (v.g. el abordaje de fuentes históricas con preguntas antropológicas), y d) el nombre mismo es cuestionado por separar unas historias de otras.

En el caso andino, la Arqueología jugó un papel tan importante como la Historia y la Antropología. De acuerdo a los desarrollos locales, se propusieron otras denominaciones -como Historia andina o Antropología histórica- para sustituir el término inicial y compartido de Etnohistoria. Salvo en Chile, no se generaron espacios de formación de etnohistoriadores (Chiappe, 2018), ni de grado ni de posgrado, y no existió un órgano de difusión de las investigaciones exclusivo de la Etnohistoria andina. Finalmente, la adscripción y autoadscripción de los investigadores varía con el correr de los años.

Por lo tanto -como plantea el eje central de este apartado-, si intentaba limitar mi indagación a trabajos que se definieran explícitamente como etnohistóricos, quedaban afuera múltiples referentes compartidos y la Etnohistoria se diluía hasta casi desaparecer. Para no quedar atrapada en la idea de crisis ni tratar los usos actuales del término como relictos del pasado, era necesario que considerara investigadores, encuentros, publicaciones y equipos institucionales que no siempre eran definidos como "etnohistóricos" a lo largo de todo el periodo. Pero, entonces, ¿cómo abrir el espectro y bajo qué criterios? En los siguientes apartados 
presentaré de qué manera abordé este problema. En primer lugar, haré referencia a la selección de los materiales de análisis y luego indicaré cómo de delimité el objeto de mi indagación.

\section{Criterios PARA LA SELEcción DE LOS MATERIALES DE ANÁLISIS}

El material analizado consistió en entrevistas, observaciones y fuentes documentales. Combiné el relevamiento de fuentes en archivos institucionales y personales con la participación en eventos académicos, encuentros informales y entrevistas a investigadores y a referentes institucionales en Buenos Aires, Lima, Cusco, La Paz y Sucre. El foco del análisis estuvo puesto en los documentos, ya que el material de entrevistas y observaciones fue utilizado de manera auxiliar y complementaria preferentemente para identificar investigadores, instituciones, publicaciones, temas o formas de financiación y a partir de allí realizar el rastreo documental. En este sentido, el acceso a los documentos es resultado -en buena medida- de la densidad de los lazos establecidos en la investigación. Se trata de un acceso mediado por personas, no solo por aquellas encargadas de su conservación, sino por quienes pueden facilitar o dificultar el acceso a través de contactos ( cfr. Muzzopappa y Villata, 2010; Schijman, 2010). ${ }^{3}$

El análisis del desarrollo de la Etnohistoria andina a partir de las prácticas de investigación, como he dicho, exigía identificar equipos de investigación y redes académicas, seguir sus publicaciones y participaciones en eventos académicos e indagar en la gestión de espacios institucionales. Sin embargo, primero debía resolver cómo elegir, qué grupos y qué trayectorias seguir, teniendo en cuenta los desafíos de delimitación antes indicados. Para ello desarrollé dos estrategias: en primer lugar, reconstruí la red en torno a una figura considerada central para el desarrollo de la Etnohistoria andina; en segundo lugar, una vez reconstruida esta red, identifiqué los principales espacios de intercambio y debate en los que participaron esos investigadores, y analicé sus controversias.

John Murra fue uno de los principales gestores de una red internacional e interdisciplinar de investigadores que combinó lazos anclados institucionalmente con otros de carácter más informal. Esta red se constituyó en un nodo central de la red conformada por la comunidad internacional de investigadores dedicados a los estudios andinos. Logró captar la atención de investigadores con diferentes intereses y generar un marco común de debate gracias a la plasticidad y la capacidad de aglutinación de su propuesta teórico-metodológica para estudiar el mundo andino. A esto se suma su peregrinaje por distintos centros académicos de la región y la organización de seminarios informales que permitían exponer y compartir las investigaciones en progreso. Murra utilizó la correspondencia para reforzar los lazos personales y para poner en circulación artículos, tesis y fuentes inéditas. Muchos de estos materiales lograron ser publicados gracias a sus gestiones, que traspasaban las fronteras nacionales. Esta movilidad puede ser apreciada también a partir de los cursos de posgrado que dictó en los distintos países que visitó, y del empleo estratégico de los recursos otorgados por la Fundación Ford, la Comisión Fulbright o el Institute of Andean Research, a través de becas, seminarios internacionales y proyectos interdisciplinares. La reconstrucción y el análisis de la "comunidad andina”, que Murra impulsó, fueron presentados en un trabajo previo (Ramos, 2015), que combinaba un enfoque de redes (Devés, 2007) con la propuesta de biografías colectivas (Ruiz Torres, 2010).

La referida reconstrucción me llevó a indagar en fuentes que me permitieran seguir a estos investigadores, no de manera individual sino a través tanto de sus interlocuciones en encuentros presenciales con distinto grado de institucionalización como de sus escritos. El corpus quedó compuesto por compilaciones temáticas y de homenaje, actas de congresos y jornadas, números temáticos y de debate de las principales revistas, entrevistas publicadas, memorias institucionales, proyectos de investigación y cartas. Para complementar lo anterior realicé entrevistas a media centena de investigadores en los tres países de interés. En ellas indagué en aspectos específicos de la práctica profesional, como la propia percepción de su labor, los referentes académicos que consideraban ineludibles y los espacios institucionales de pertenencia. 
Todo lo anterior me permitió trascender la red de investigadores, que había reconstruido inicialmente a partir de la figura de Murra, ya sea porque en los espacios de encuentro aparecían de manera reiterada otros nombres o porque me los sugerían en las entrevistas. Fue así que trabajé con equipos específicos que transitaron por los siguientes espacios institucionales: Pontificia Universidad Católica del Perú, Universidad Nacional de San Antonio de Abad del Cusco, Universidad Nacional de San Cristóbal de Huamanga, Universidad Mayor de San Andrés, Universidad de Buenos Aires, Instituto de Estudios Peruanos, Instituto Francés de Estudios Andinos.

Fui rastreando distintas maneras de delimitar la Etnohistoria andina y los principales conceptos asociados a ella a partir de la documentación relativa a la organización de eventos académicos. A modo de ejemplo, en 1976 se organizaron las Primeras Jornadas del Museo Nacional (Perú) con el objetivo de colaborar con el desarrollo de los estudios etnohistóricos. Dos años después, cuando se publicaron las memorias, se dejó en claro que solo se incluyeron las ponencias que "tratan los temas relacionados más estrechamente con la definición de Etnohistoria adoptada por el comité organizador", es decir, "la historia de los pueblos ágrafos del área andina cuyo desarrollo cultural podría ser detectado en los documentos de los siglos XVI y XVII”. Esto dejaba fuera las presentaciones que trataron "temas actuales" (Paredes y Castelli, 1978, pp. 9-10). Esta fue la primera referencia que encontré que me permitía revisar la idea generalmente aceptada de que el corrimiento de la etnohistoria hacia temporalidades más recientes era posterior a la década del 80, y que se había dado en otros países de la región andina pero no en Perú. ${ }^{4}$

Tras identificar las principales revistas y editoriales en las que los etnohistoriadores publicaban su producción fui reconstruyendo los temas más convocantes y los principales debates, tema al que volveré sobre en el apartado siguiente. Una vez delimitados los equipos pude rastrear las formas de financiación, la formación de discípulos y el impulso que estas personas dieron a la creación de institutos de investigación, revistas y encuentros de especialistas. ${ }^{5}$ Por otra parte, la articulación entre las entrevistas y el examen de documentos me permitió reconstruir un episodio en el cual se puso en duda la autoría de una fuente documental (crónica), hecho que conmocionó a la comunidad académica, al reeditar discusiones sobre el lugar de los especialistas, la honestidad intelectual y el crédito académico. ${ }^{6}$ De esta manera, fui reconstruyendo lo que Knorr Cetina (1996) denominó como la contextualidad de la acción científica, la cual atraviesa las formas de producir y nos permite aproximarnos a comprender el modo en que se van delineando las fronteras de la especialidad.

\section{CONSTRUCCIÓN DEL OBJETO DE INVESTIGACIÓN Y RESULTADOS DE LA INDAGACIÓN}

Inicialmente vislumbré la posibilidad de seleccionar y seguir el desarrollo de ciertos debates en las investigaciones de Etnohistoria andina, por ejemplo, aquellos sobre ayllus, comunidad, estado, rebeliones o mestizaje. Sin embargo, en estos la Etnohistoria andina aparecía diluida, ya que las discusiones no eran específicamente etnohistóricas. Por ejemplo, el caso del ayllu implicaba adentrarse en la vasta tradición antropológica de estudios de parentesco (c. Bossert et al., 2012), o de la Historia para rebeliones (c. Serulnikov, 2012), y así en cada caso.

Sin embargo, tras una primera exploración de estos debates, el intento fallido me permitió notar que había un hilo conductor en la manera en que algunos investigadores participaban de las discusiones. Este tenía que ver con cómo entendían "lo andino", ya que participaban de los debates recuperando sentidos de lo andino como parte central de la discusión que daban. La idea de "lo andino", que era retomada por investigadores de distinta procedencia y formación -incluso con orientaciones teórico-metodológicas opuestas-, permitía entonces delimitar una comunidad con intereses compartidos. Examinar la Etnohistoria desde esta perspectiva me permitió identificar intercambios que atravesaban todo el periodo de análisis y revisar la paradoja de presencia/ausencia señalada en el punto 3. 
A partir de entonces indagué los elementos que a lo largo del siglo XX aportaron a los sentidos de "lo andino”, y que, de forma articulada o superpuesta, fueron sedimentándose con el tiempo ${ }^{7}$ (Ramos, 2018; Gade, 1999). Identifiqué luego dos hitos vinculados estrechamente a la Etnohistoria: el logro andino y la crítica al andinismo. El primero, expresado en los grandes modelos de acceso a recursos (archipiélagos verticales (Murra, 1972), costero (Rostworowski, 1977), de pastores ( $(f r$. Medinacelli 2005)) y en la diversificación o atomización de lo andino (Martinez 2005). El segundo, atravesado por las discusiones sobre las características de la expansión incaica (fronteras del imperio), las implicancias del mestizaje (Salomon, 1994; Saignes, 1990) y la relación de las sociedades contemporáneas con el pasado de la región (Mayer 1991). La fluidez que adquirió mi investigación a partir de ese momento me llevó a proponer que la identificación de hilos conductores de este estilo resulta eficaz para analizar el devenir de otros campos de investigación, ya sea que se tratare de disciplinas o de espacios interdisciplinares.

Una vez delimitado de esta manera el corpus a considerar - evitando definiciones normativas sobre qué es o debiera ser la Etnohistoria andina y partiendo de las prácticas colectivas y situadas-, identifiqué cuatro elementos estructurantes a lo largo del periodo analizado: 1) el alcance geográfico, 2) la temporalidad, 3) la dimensión étnica, y 4) las voces de los sujetos en los documentos. Con elementos estructurantes me refiero a que se debe tener en cuenta a todos ellos para reconstruir el desarrollo de este campo de estudios. En tanto las referencias empíricas que permiten sostener esta afirmación se encuentran presentadas en Ramos (2016b, 2018), me limitaré en lo siguiente a sintetizar de qué manera cada uno de los elementos mencionados se resignificaron a lo largo del período.

En lo que respecta al alcance geográfico, se partió de una crítica al cuscocentrismo. Aunque, a medida que se avanzaba sobre las "zonas periféricas" se creaban nuevos márgenes para explorar. Así, del cuestionamiento al cuscocentrismo se pasó al serranocentrismo, y luego al andinocentrismo. Tal ampliación regional, en principio de la mano de las formas de control incaicas pero cada vez más en sus propios términos, llevó a que las excepciones a un modelo unívoco de "lo andino" comenzaran a ser cada vez más recurrentes (Ramos, 2018).

Desde la Etnohistoria se instaló la imagen, propiciada por la articulación disciplinar propugnada, de un continuum andino que conectaba las sociedades prehispánicas con las contemporáneas. Las implicancias políticas de las delimitaciones espaciales arqueológicas e históricas fueron vertientes que establecieron un vínculo con el presente. Otra fue la revisión de conceptos centrales en las investigaciones, como ayllu y comunidad, que requirieron de una aproximación histórica y comparativa que permitiese reconstruir sus sentidos y transformaciones, a fin de evitar una mirada esencialista de las manifestaciones actuales (Ramos 2016b).

Los sujetos fueron inicialmente definidos desde la etnicidad. Se trataba de hacer la historia de aquellos grupos conquistados por los incas y luego por los españoles. En la misma línea, la revalorización de la complejidad de la organización social de las poblaciones contemporáneas -en los tiempos de la modernización y el desarrollismo- mantuvo el foco en la etnicidad. Las ideas de cambio y de continuidad se fueron vinculando con distintos énfasis, y el avance en las investigaciones impuso la idea de un mundo y sujetos heterogéneos que requerían un distanciamiento de las lecturas binomiales. Si por un lado el concepto de "andinos" se hizo más amplio que el de "indígenas", por el otro se profundizó en una necesaria articulación con las dimensiones de clase y género. La idea de mestizaje cultural, que se remonta a inicios de los 90, abrió una nueva senda en las investigaciones (Ramos, 2016b).

En lo referente a las voces "andinas" y los documentos, al partir la Etnohistoria andina de una mirada descentrada -en principio, al menos de Cusco-, su puesta en práctica fue de la mano de una preocupación metodológica: ¿dónde encontrar esas otras voces? Visitas, litigios, textos literarios fueron las primeras alternativas. A medida que avanzaron las investigaciones las voces se tornaron cada vez más polifónicas y se profundizó el desafío metodológico a partir de la consideración de los distintos sistemas de soporte. Paralelamente, se desarrolló una revisión de la manera en que se concebían las fuentes, que implicó un 
distanciamiento de la idea de reflejo de la realidad y un desplazamiento hacia el interés por los procesos de producción de sentido a través de los cuales se inscribe la información (Ramos, 2018).

Tras reconstruir de esta manera el desarrollo de la Etnohistoria andina, propuse que -a pesar de las transformaciones en las investigaciones- era posible sostener que las coordenadas desde las cuales se realizan las preguntas atraviesan todo el periodo de análisis. Desde las indagaciones iniciales se consideró que era necesario renovar las preguntas sobre las sociedades andinas, y para eso se debía: 1) correr el foco de las fuentes habituales; 2) evitar la imposición de categorías ajenas (como socialismo, esclavismo, etc.) y, en su lugar, recuperar las formas propias de las sociedades estudiadas; 3) adoptar un enfoque comparativo e interdisciplinario (Ramos, 2016b).

\section{CONSIDERACIONES FINALES}

Si hacemos una síntesis de las ideas expuestas, se puede afirmar que el estudio de la producción y circulación del conocimiento científico plantea desafíos que son comunes más allá de un objeto particular. Estos temas se han tratado desde distintas tradiciones de investigación, cuyos aportes es posible retomar y articular con la propia tradición antropológica. Me he inclinado por la toma de distancia de definiciones normativas, con la consecuente caracterización a partir de la indagación en prácticas colectivas y situadas. Esto resultó particularmente operativo en un campo de límites difusos al cual muchos de sus integrantes no consideran una disciplina. Sin embargo, considero que es deseable una aproximación de este tipo incluso cuando el objeto de estudio se presenta como más claramente delimitado.

Los desafíos de delimitación que emerjan al inicio de la investigación producto de la apertura señalada, una vez superados, conducirán a una lectura más comprehensiva de los procesos estudiados. Para ello, resulta fundamental reconstruir las interacciones entre investigadores e identificar conceptos que constituyen un verdadero hilo conductor entre los distintos temas que se abordan en el campo estudiado y que tengan a su vez una continuidad en el tiempo. Una vez identificado un concepto central, se deben explorar los principales sentidos que condensa -en muchos casos contrapuestos- y establecer cuáles son los principales temas de debates que atraviesa.

Propongo entonces arribar a una caracterización del campo estudiado a partir de la identificación de: 1) las formas de interacción entre los investigadores, 2) un concepto-hilo conductor, 3) los temas que estructuran el devenir de las investigaciones, y 4) las coordenadas que perfilan las preguntas de los investigadores. En el caso tratado esto me permitió sostener que aspectos como la fragmentación de lo andino, la crítica a la división entre indígenas y no indígenas, y el cuestionamiento a enfoques esencialistas -que suelen ser asociados a la crisis de la Etnohistoria y producto de cuestionamientos externos al campo- son parte constitutiva de su desarrollo. Al fundamentar que la revisión continua de los límites espaciales, temporales y conceptuales caracterizó a la Etnohistoria andina y cómo esto condujo -en sucesivas oportunidades- a la formulación de nuevas preguntas, fue posible ofrecer otra interpretación del desarrollo de este campo que no tratara las investigaciones contemporáneas como relictos del pasado.

Como producto del trabajo realizado, luego de haber accedido a un panorama general del desarrollo de las investigaciones en Etnohistoria andina, se abrieron una serie de líneas de indagación:

a) ¿Cómo se sostiene en la práctica el enfoque interdisciplinario y trasnacional y cuán significativas resultan las redes informales para ello? En relación a ello se deprenden desafíos específicos del tipo de documentos (cartas, homenajes, agradecimientos) en los cuales rastrear dichos vínculos.

b) ¿Cuáles son las potencialidades de entender "lo andino" como un concepto (sensu Koselleck, 2004) y el entramado semántico del que forma parte? Para ello debe profundizarse su articulación con otros conceptos que llevan implícita la idea de área -como Mesoamérica o Amazonía-, pero también aquellos como marginalidad y desarrollo que son recurrentes en las publicaciones. 
c) ¿De qué manera se puede trascender el ámbito académico para avanzar en una interpretación más compleja de las formas en que devienen campos de investigación? Para ello podrían abordarse, por ejemplo, las características de las academias nacionales en relación a cómo desde cada país se ha concebido a indígenas y mestizos. A su vez, esto abre el camino para considerar las apropiaciones y las influencias de las organizaciones sociales en la producción académica.

Dichas líneas de indagación dan cuenta de los caminos que comencé a transitar una vez finalizada la investigación doctoral. He querido mencionarlas para referir no solo los resultados del enfoque implementado sino también las nuevas preguntas que generó, y dar cuenta de manera más completa los alcances de las decisiones teórico-metodológicas aquí exploradas. En contraste con las miradas que parten de una definición normativa de la disciplina estudiada, un análisis de este tipo gana en profundidad y abre la mirada a relaciones transversales, alentando a trazar vínculos con procesos sociopolíticos a distinta escala y conexiones que atraviesan los campos disciplinares.

\section{Agradecimientos}

Agradezco a los evaluadores anónimos por los comentarios precisos y los aportes que enriquecieron el texto, y a Carlos Zanolli y Carlos Chiappe por la lectura crítica de una versión preliminar de este trabajo.

\section{REFERENCIAS}

Arêas Peixoto, F. (2008). El diálogo como forma: antropología e historia intelectual. Prismas, 12, 17-32. Recuperado de http://www.scielo.org.ar/scielo.php?script=sci_arttext\&pid=S1852-04992008000100001\&lng=es\&tlng=es.

Bosa, B., y Santoyo, A. (2010). Etnografía y archivos. Revista Colombiana de Antropología, 46(2), 243-248. Recuperado de http://www.scielo.org.co/scielo.php?script=sci_arttext\&pid=S0486-65252010000200001\&ln $\mathrm{g}=\mathrm{en} \& \mathrm{t} \operatorname{lng}=\mathrm{es}$.

Bossert, F., Sendón, P., y Villar, D. (2012). Elparentesco: textos fundamentales. Buenos Aires: Biblios.

Cardoso De Oliveira, R. (1999/2000). Peripheral Anthropologies “versus" Central Anthropologies. Journal of Latin American Anthropology, 4-5 (2-1), 10-30.

Castorina, J. (1989). La posición del objeto en el desarrollo del conocimiento. En J. Castorina, B. Aisenberg, D. Dibar Ure, G. Collinvaux y G. Palau (Comps.), Problemas en Psicología Genética (pp. 37-62). Buenos Aires: Miño y Dávila.

Chiappe C. (2018). Entre las luchas pasadas y las presentes. Antecedentes, surgimiento y desarrollo de la etnohistoria andina chilena (Tesis de doctorado en Antropología). Universidad de Buenos Aires, Buenos Aires, Argentina.

Chiappe, C., y Ramos, A. (2017). Estrategias de búsqueda y sistematización de fuentes escritas. En A. Domínguez Mon (Ed.), Trabajo de campo etnográfico: prácticas y saberes. Metodología y Técnicas de la Investigación de Campo (pp. 23-52). Buenos Aires: EFFL. Recuperado de http://publicaciones.filo.uba.ar/sites/publicaciones.filo.uba.ar/fil es/Trabajo\%20de\%20campo\%20etnogr\%C3\%A1fico_0.pdf

Chiappe, C., y Ramos, A. (2016). Caja de resonancia: el papel de los encuentros académicos en una época de polarización política. Revista de Ciencias Sociales, 37, 71-90. Recuperado de https://www.redalyc.org/pdf/708 170849360005.pdf

Devés, E. (2007). Redes intelectuales en América Latina: Hacia la constitución de una comunidad intelectual. Chile: Instituto de Estudios Avanzados de la Universidad de Santiago de Chile.

Dube, S. (2007). Antropología, historia y modernidad. Cuestiones críticas. Estudios de Asia y África, 42(2), 299-337. Recuperado de http://www.uacm.kirj.redalyc.redalyc.org/articulo.oa?id=58611171002

Escobar, A. (2007). La invención del Tercer Mundo Construcción y deconstrucción del desarrollo. Caracas: Fundación Editorial el perro y la rana.

Gadamer, H. (1988). Verdad y método. Salamanca: Sígueme. 
Gerholm, T. \& Hannerz, U. (1982). Introduction: The Shaping of National Anthropologies. Ethnos, 47, 5-32.

Gil, G. (2016). Producción teórica y circulación de ideas en las ciencias sociales en la Argentina. Tres casos contrastantes de las décadas de 1960 y 1970. Cuadernos de Antropología Social, 43, 51-71. Recuperado de: https://www.reda lyc.org/pdf/1809/180948645006.pdf

Hidalgo, C., y Stagnaro, A. (2016). Antropología de la Ciencia y la Tecnología. Cuadernos de Antropología Social, 43, 9-11. Recuperado de: http://www.scielo.org.ar/pdf/cas/n43/n43a01.pdf

Knorr Cetina, K. (1996). ¿Comunidades científicas o arenas transepistémicas de investigación? Una crítica de los modelos cuasi-económicos de la ciencia. Redes, 3(7), 129-160. Recuperado de: http://ridaa.unq.edu.ar/bitstrea $\mathrm{m} /$ handle/20.500.11807/671/08R1996v3n7.pdf?sequence $=1$ \&isAllowed $=\mathrm{y}$

Koselleck, R. (2004). Historia de los conceptos y conceptos de historia. Ayer , 53, 27-45. Traducción de Javier Fernández Sebastián y Gonzalo Capellán de Miguel. Recuperado de https://www.ahistcon.org/PDF/numeros /ayer53_HistoriaConceptos_Fernandez_Fuentes.pdf

Krotz, E. (1997). Anthropologies of the South: Their Rise, Their Silencing, Their Characteristics. Critique of Anthropology, 17(3), 237-251.

Martínez, J. (2005). La construcción de identidades y de lo identitario en los estudios andinos (para continuar un debate). Actas del Primer Simposio de Pensamiento Andino. Cuenca: UNESCO-Banco Central del Ecuador.

Mayer, E. (1991). Peru in Deep Trouble: Mario Vargas Llosa's 'Inquest in the Andes' Reexamined. Cultural Anthropology, 6(4), 466-450.

Medinacelli, X. (2005). Los pastores andinos: una propuesta de lectura de su historia. Ensayo bibliográfico de etnografía e historia. Boletin del IFEA, 34(3), 463-474.

Moro Abadía, O. (2005). La nueva historia de la ciencia y la sociología del conocimiento científico: un ensayo historiográfico. Asclepio, LVII(2), 255-280. Recuperado de: http://asclepio.revistas.csic.es/index.php/asclepio/ article/view/66/68

Murra, J. (1972). El "control vertical” de un máximo de pisos ecológicos en la economía de las sociedades andinas. En J. Murra, (Ed.), Visita a la provincia de León de Huánuco en 1562 por Iñigo Ortiz de Zúñiga II (pp. 427-476). Huánuco: Universidad Nacional Hermilio Valdizan.

Muzzopappa, E., y Villata, C. (2011). Los documentos como campo. Reflexiones teórico-metodológicas sobre un enfoque etnográfico de archivos y documentos estatales. Revista Colombiana de Antropología, 47(2), 13-42. Recuperado de http://www.scielo.org.co/pdf/rcan/v47n1/v47n1a02.pdf

Palti, E. (2007). La Nueva Historia Intelectual y sus repercusiones en América Latina. História Unisinos 11(3), 297305.

Platt, T. (2013). Entre la rutina y la ruptura. El archivo como acontecimiento de terreno. En C. Zanolli, J. Costilla, D. Estruch y A. Ramos (Comps.), Los estudios andinos hoy: práctica intelectual y estrategias de investigación (pp. 217-242). Rosario: Prohistoria.

Prismas, Consejo de Dirección. (2007). Presentación al Dossier Encuesta sobre historia intelectual. Prismas, 11, 151.

Ramos, A. (2011). La Etnohistoria andina antes de su consolidación. Confluencias disciplinares y propuestas teóricometodológicas. Buenos Aires: Sociedad Argentina de Antropología.

Ramos, A. (2015). El aporte de J. Murra al desarrollo de una red académica trasnacional de estudios sobre el mundo andino. Cuadernos INAPL, 24(2), 98-115.

Ramos, A. (2016a). Etnohistoria(s): contextos de emergencia y vigencia discutida. Relaciones $S A A$, 41(1), 15-34. Recuperado de http://www.saantropologia.com.ar/wp-content/uploads/2016/08/01-Ramos.pdf

Ramos, A. (2016b). El desarrollo de la Etnobistoria andina como campo interdisciplinar: interacciones entre Historia, Arqueología y Antropología (Perú, Bolivia y Argentina, 1970-2005) (Tesis de doctorado en Antropología). Universidad de Buenos Aires, Buenos Aires, Argentina.

Ramos, A. (2018). El desarrollo de la Etnohistoria andina a través de la (re)definición de lo andino (1970-2005). Fronteras de la Historia, 23(2), 8-43. Recuperado de: blob:http://www.icanh.gov.co/a5269160-617a-45a9-aa5 6-d3b3eeb9acca 
Ramos, A., y Chiappe, C. (2018a). En la Trama de la Etnohistoria Americana. Tarija: La Pluma del Escribano.

Ramos, A., y Chiappe, C. (2018b). El concepto de estrategia y la Nueva Etnohistoria. Manuscrito. Buenos Aires

Restrepo, E. (2012). Antropologías Disidentes. Cuadernos de Antropología Social, 35, 55-69. Recuperado de http:// www.scielo.org.ar/scielo.php?script=sci_arttext\&pid $=$ S1850-275X2012000100004

Ribeiro, G., y Escobar, A. (Eds.) (2008). Antropologias del mundo: Transformaciones disciplinarias dentro de sistemas de poder. México: Wenner-Gren Foundation; Ciesas. Recuperado de http://www.ciesas.edu.mx/publicaciones /clasicos/libros/Antropologias_del_mundo.pdf

Rostworowski, M. (1977). Etnia y sociedad. Costa peruana pre-hispánica. Lima: Instituto de Estudios Peruanos.

Ruiz Torres, P. (2010). Biografía e Historia. Red "teoría y práctica de la biografía". Le singulier et le collectif à l'épreuve de la biographie. College d'espagne. Recuperado de http://www.valencia.edu/retpb/docs/Texto\%20Ruiz.pdf.

Saignes, T. (1990). ¿Es posible una historia "chola" del Perú? (Acerca de Nacimiento de una utopía de Manuel Burga). Allpanchis, XXII 35/36(2), 635-657.

Salomon, F. (1994). La textualización de la memoria en la América andina: Una perspectiva etnográfica comparada. América indigena, LIV(4), 229-272.

Schijman, E. (2010). Etnografía de archivos administrativos. Un corpus de demandas y quejas menores en un barrio de vivienda social. Revista Colombiana de Antropología, 46(2), 279-305. Recuperado de http://www.scielo.or g.co/scielo.php?script=sci_arttext\&pid=S0486-65252010000200003\&lng=es\&tlng=es

Serulnivok, S. (2012). La Insurrección Tupamarista: Historias e Historiografías. 20/10 El mundo atlántico y la modernidad iberoamericana, 1750-1850, pp. 251-264. Recuperado de: http://www.20-10historia.com/articulo 14.phtml

Stocking, G. (1982). On the limits of 'presentism' and 'historicism' in the historiography of the behavioral sciences. En Race, culture and evolution. Essays in the History of Anthropology, pp.1-12. Chicago/Londres: The University of Chicago Press.

\section{Notas}

1 Esta propuesta es consistente con el modelo de construcción de conocimiento al que adhiero: el constructivismo crítico propuesto por la epistemología genética. Los representantes de esta teoría sostienen que el conocimiento se produce mediante la interacción entre el sujeto y el objeto, a través de la acción significativa por la cual el primero transforma al segundo al actuar sobre él y al hacerlo se transforma a sí mismo, de manera que cada avance en el conocimiento da lugar a nuevas preguntas. Al sostener una aproximación continua entre objeto de conocimiento construido y la realidad, la epistemología genética rechaza la filosofía escisionista -tanto el empirismo filosófico como el apriorismo-, toda vez que se opone a la mera reproducción al mismo tiempo que reconoce una adecuación del sistema cognoscitivo, proponiendo un realismo crítico que implica la existencia de lo real y una tendencia a la objetividad -concebida en tanto horizonte, nunca alcanzable completamente (Castorina, 1989)-

2 Los tres investigadores considerados como fundadores de la etnohistoria andina son John Rowe, John Murra y Tom Zuidema. Mi investigación para la tesis de licenciatura se ocupó de los dos primeros.

3 Dicho foco en los documentos, en el marco de una investigación antropológica, me condujo a revisar las discusiones y reflexiones sobre la forma en que los antropólogos trabajan con las fuentes escritas. Si bien puede parecer que se trata de algo ajeno a la Antropología, lo cierto es que -tanto en su vertiente clásica como a partir de lo que en la década de 1980 se denominó "giro histórico" - los antropólogos han empleado documentos y han expresado distintas propuestas para su tratamiento. Dado que he trabajado este punto en otra publicación (Cfr. Chiappe y Ramos, 2017), no me detendré aquí en las propuestas; baste señalar que se enmarcan en un renovado vínculo entre Antropología e Historia (Dube, 2007), y que los antropólogos han argumentado acerca de la posibilidad de considerar el trabajo de archivo como trabajo de campo (Nacuzzi, 2002), de pensar el archivo como acontecimiento de terreno (Platt, 2013) o realizar una etnografía de los archivos (Bosa y Santoyo, 2010). Investigaciones de este tipo se han nutrido tanto de la memoria como de distintos tipos de repositorios, y de documentación que no necesariamente se encuentra archivada.

4 Este es solo un ejemplo pequeño del material de eventos académicos trabajado en la investigación doctoral. En Ramos y Chiappe (2018a) puede encontrarse un análisis detallado a partir de los documentos relativos a la organización del I Congreso Internacional de Etnohistoria. Asimismo, se encuentra en evaluación un artículo que focaliza en los sentidos que los etnohistoriadores andinos le daban al concepto de estrategia en la década de 1980. En dicho manuscrito se analiza 
el carácter complementario de dos tipos de documentación: publicaciones que aplican el concepto y cartas donde la organizadora de un congreso y el coordinador de un simposio que lleva en nombre "estrategias" discuten su alcance y aplicación (Ramos y Chiappe, 2018b).

5 La mayor parte de estos avances no han sido publicados aún; se encuentran solo en mi tesis doctoral. Sin embargo, en Ramos (2015) hay algunas referencias específicas a las actividades de John Murra en este sentido, en Zanolli et al. (2010) se tratan en detalle estos elementos en relación a un equipo de la Universidad de Buenos Aires, y en Chiappe y Ramos (2016) se encontrarán referencias a cómo la creación de espacios de posgrado se vio atravesada por la marcada polarización política de la década de 1960.

$6 \mathrm{Al}$ concluir el IV Congreso Internacional de Etnohistoria (1996), Laura Laurencich Minelli comunicó que había localizado en Nápoles un códice que revelaba que el verdadero autor de la Nueva Corónica y buen gobierno era el jesuita Blas Valera y no Guaman Poma. Ofreció publicar el códice y un análisis al Journal de la Societe des Americanistes de París; los editores contactaron a especialistas para que emitieran su opinión y el texto finalmente no fue aceptado para su publicación. La investigadora difundió entonces su hallazgo a través de la prensa y buscó editarlo en Perú y en Italia. Como consecuencia, Juan Carlos Estenssoro decidió hacer pública su evaluación, en la que cuestionaba fuertemente la autenticidad del documento. Lo ocurrido en el congreso solo pudo ser reconstruido a partir de las entrevistas; luego se identificaron 5 textos publicados en 1998 por investigadores vinculados a la etnohistoria andina que se pronuncian en relación a la polémica.

7 Por ejemplo, espacio andino no es una delimitación geográfica que existe objetivamente más allá de las negociaciones de sentido entre múltiples actores; distintos elementos son recuperados y esgrimidos como sostén de los argumentos en pos de una definición más amplia o más restringida. La división de países que forman parte del Pacto Andino y del ABC tiene incidencia sobre cómo se piensan los alcances de las propuestas de trascender las fronteras nacionales. Estas formas de pensar la unidad regional tienen eco asimismo en la conformación de las instituciones académicas (considerarse, por ejemplo, la delimitación que establece el Instituto Francés de Estudios Andinos). Una lúcida exploración de la imbricación de sentidos asignados y agentes que reclaman lo andino puede encontrarse en Martínez (2005). 